\title{
Fixed point theorems in constructive mathematics
}

\author{
MATTHEW HENDTLASS
}

\begin{abstract}
This paper gives the beginnings of a development of the theory of fixed point theorems within Bishop's constructive analysis. We begin with a constructive proof of a result, due to Borwein, which characterises when some sets have the contraction mapping property. A review of the constructive content of Brouwer's fixed point theorem follows, before we turn our attention to Schauder's generalisation of Brouwer's fixed point theorem. As an application of our constructive Schauder's fixed point theorem we give an approximate version of Peano's theorem on the existence of solutions of differential equations. Other fixed point theorems are mentioned in passing.
\end{abstract}

2010 Mathematics Subject Classification 03F55, 03F60, 46S30 (primary); 34A30, $47 \mathrm{H} 10$ (secondary)

Keywords: constructive analysis, fixed point theorems, differential equations

\section{Introduction}

Fixed-point theorems are a major tool in both functional analysis and mathematical economics ${ }^{1}$ and are used to prove the existence of solutions to differential equations and the existence of Nash equilibria among other things. Despite this, the constructive literature on fixed point theorems has been scant ${ }^{2}$. There are (at least) two reasons for this:

(i) The standard proof of the simplest and most useful of the well known fixed point theorems, the Banach fixed point theorem, is essentially constructive.

(ii) The non-constructive nature of Brouwer's fixed point theorem, and the subsequent rejection of this theorem by Brouwer, is well known, and a constructive approximate version for simplices (via Sperner's lemma) is part of the folklore.

\footnotetext{
${ }^{1}$ The main fixed point theorem used in mathematical economics, Kakutani's fixed point theorem, is given a constructive treatment in [16].

${ }^{2}$ Although [9] gives a Bishop-style constructive treatment of Edelstein's fixed point theorem; and Kohlenbach [18, Chapter 18] examines contractive and nonexpansive fixed point theorems for computational content using tools from proof theory.
} 
Only recently has a fully constructive proof of the approximate version of Brouwer's fixed point theorem, for simplices, been presented [23].

By constructive mathematics we mean mathematics done in the framework of Bishop's constructive mathematics, which is essentially mathematics with intuitionistic logic and dependent choice ${ }^{3}$. Working with intuitionistic logic ensures that proofs proceed in a manner which preserves computational meaning; in particular, a constructive proof of $\exists_{x} P(x)$ embodies an algorithm for the construction of an object $x$ and an algorithm verifying that $P(x)$ holds. In this manner, constructive mathematics can be viewed as a high-level programming language. It should be noted that the constructive mathematician is interested only in what can in theory be computed, and is not concerned with questions of practicality.

This paper is broken up into three sections. In the first we consider the fixed point theorems of Banach and Brouwer; we prove that spaces with a strong connectedness property are complete if and only if every contraction mapping has a fixed point, and we give an approximate version of Brouwer's fixed point theorem for uniformly sequentially continuous functions on totally bounded subsets of $\mathbf{R}^{n}$ with convex closures. The second section presents a constructive treatment of Schauder's fixed point theorem and its extension due to Rothe. In the final section we give an application of our constructive Schuader's fixed point theorem: we prove an approximate version of Peano's theorem on the existence of solutions of differential equations.

For convenience, we pause here to recall some constructive definitions. Let $X$ be a metric space and let $S$ be a subset of $X$. If there exists $x \in S$, then $S$ is said to be inhabited; constructively this is a stronger property than $S$ being nonempty, $\neg(S=\emptyset)$. An inhabited set $S$ is said to be located if for each $x \in X$ the distance

$$
\rho(x, S)=\inf \{\rho(x, s): s \in S\}
$$

from $x$ to $S$ exists. Let $\varepsilon>0$. An $\varepsilon$-approximation to $S$ is a subset $T$ of $S$ such that for each $s \in S$, there exists $t \in T$ such that $\rho(s, t)<\varepsilon$. We say that $S$ is totally bounded if for each $\varepsilon>0$ there exists a finitely enumerable ${ }^{4} \varepsilon$-approximation to $S$. A metric space is said to be compact if it is complete and totally bounded. A totally bounded subset of $X$ is located [4, page 95, Propostion (4.4)].

\footnotetext{
${ }^{3}$ See $[4,10,12]$ for an introduction to constructive mathematics, and see $[1,5,14,19]$ for constructive alternatives to ZFC.

${ }^{4} \mathrm{~A}$ set is finitely enumerable if it is the image of $\{1, \ldots, n\}$ for some $n \in N^{+}$, and a set is finite if it is in bijection with $\{1, \ldots, n\}$ for some $n \in N^{+}$; constructively these notions are distinct.
} 


\section{The two main fixed point theorems}

\subsection{Banach's fixed point theorem}

A mapping $f$ from a metric space $X$ into itself is said to be a contraction mapping if there exists a contraction constant $r \in(0,1)$ such that

$$
\rho(f(x), f(y))<r \rho(x, y)
$$

for all $x, y \in X$. A point $x \in X$ is a fixed point of $f$ if $f(x)=x$, and a metric space $S$ is said to have the contraction mapping property if every contraction mapping from $S$ into $S$ has a fixed point. Any fixed point of a contraction mapping is necessarily unique, and if a metric space $S$ has the contraction mapping property, then so does its completion.

The simplest and most useful of the standard fixed point theorems is the Banach fixed point theorem: every contraction mapping of a complete metric space into itself has a fixed point. The standard proof of this theorem (see [22] or the first part of the proof of Theorem 1) is fully constructive.

We present a constructive proof of a result, due to Borwein [13], which characterises when some sets with a strong connectedness property have the contraction mapping property. A metric space $X$ is said to be uniformly Lipschitz-connected if there exists a positive real number $L$ such that for all $x, y \in X$, there exists a function $g:[0,1] \rightarrow X$ such that $g(0)=x, g(1)=y$ and

$$
\rho(g(s), g(t)) \leqslant L|s-t| \rho(g(0), g(1))
$$

for all $s, t \in[0,1]$.

Theorem 1 Let $S$ be an inhabited uniformly Lipschitz-connected metric space. Then $S$ is complete if and only if it has the contraction mapping property.

Proof Suppose that $S$ is complete. Let $f$ be a contraction mapping on $S$ with contraction constant $r \in(0,1)$, and fix $x \in S$. Let $x_{0}=x$ and $x_{n}=f^{n-1}(x)$ for each $n \in \mathbf{N}$. Then $\left(x_{n}\right)_{n \geqslant 1}$ is a Cauchy sequence in $S:$ if $m \geqslant n \geqslant 1$, then

$$
\rho\left(x_{n}, x_{m}\right) \leqslant \sum_{k=n}^{m-1} \rho\left(x_{k}, x_{k+1}\right)<\frac{r^{n}}{1-r} \rho\left(x_{0}, x_{1}\right) \longrightarrow 0 \text { as } n \longrightarrow \infty .
$$

Hence $\left(x_{n}\right)_{n \geqslant 1}$ converges to some point $x \in S$; clearly $f(x)=x$. 
Conversely, suppose that $S$ has the contraction mapping property, let $\left(x_{n}\right)_{n \geqslant 1}$ be a Cauchy sequence in $S$, and let $\hat{x}$ be the limit of $\left(x_{n}\right)_{n \geqslant 1}$ in the completion $(\hat{S}, \hat{\rho})$ of $(S, \rho)$. Without loss of generality we may take

$$
\rho\left(x_{n}, x_{m}\right)<2^{-\min \{m, n\}} \quad(m, n \in \mathbf{N}) .
$$

Using countable choice and the uniform Lipschitz-connectedness of $S$, construct $L>0$ and functions $g_{k}:[0,1] \rightarrow S(k \in \mathbf{N})$ such that for each $k, g_{k}(0)=x_{k+1}, g_{k}(1)=x_{k}$, and

$$
\rho\left(g_{k}(s), g_{k}(t)\right)<L|s-t| \rho\left(x_{k}, x_{k+1}\right)
$$

for all $s, t \in[0,1]$. Using the gluing lemma [8], define a mapping

$$
g:\{0\} \cup \bigcup_{k \geqslant 1}\left[2^{-(k+1)}, 2^{-k}\right] \cup(1, \infty) \rightarrow S \cup\{\hat{x}\}
$$

by

$$
g(t) \equiv \begin{cases}\hat{x} & \text { if } t=0 \\ g_{k}\left(2^{k+1} t-1\right) & \text { if } t \in\left[2^{-(k+1)}, 2^{-k}\right] \\ x_{1} & \text { if } t>1\end{cases}
$$

Suppose that there exist $t_{1}, t_{2}$ with $t_{2}<t_{1}$ and $\rho\left(g\left(t_{1}\right), g\left(t_{2}\right)\right)>L\left|t_{1}-t_{2}\right|$. By continuity we may assume, without loss of generality that $t_{i} \in\left[2^{-n_{i}}, 2^{-n_{i}+1}\right](i=1,2)$; set $s_{0}=t_{1}, s_{n_{1}-n_{2}}=t_{2}$, and $s_{k}=2^{-n_{2}-1+k}$ for $1 \leqslant k \leqslant n_{1}-n_{2}-1$. Then

$$
\begin{aligned}
\sum_{k=1}^{n_{1}-n_{2}} \rho\left(g\left(s_{k-1}\right), g\left(s_{k}\right)\right) & \geqslant \rho\left(g\left(t_{1}\right), g\left(t_{2}\right)\right) \\
& >L\left|t_{1}-t_{2}\right| \\
& =\sum_{k=1}^{n_{1}-n_{2}}\left|s_{k-1}-s_{k}\right|,
\end{aligned}
$$

so there exists $1 \leqslant k \leqslant n_{1}-n_{2}-1$ such that $\rho\left(g\left(s_{k-1}\right), g\left(s_{k}\right)\right)>L\left|s_{k-1}-s_{k}\right|$. But

$$
\begin{aligned}
\rho\left(g\left(s_{k-1}\right), g\left(s_{k}\right)\right) & \left.=\rho\left(g_{k}\left(2^{k+1} s_{k-1}-1\right), g_{k}\left(2^{k+1} s_{k}-1\right)\right)\right) \\
& \leqslant L 2^{k+1}\left|s_{k-1}-s_{k}\right| \rho\left(x_{k+1}, x_{k}\right) \\
& \leqslant L\left|s_{k-1}-s_{k}\right|
\end{aligned}
$$

— a contradiction. Hence

$$
\rho(g(s), g(t)) \leqslant L|s-t|
$$


for all $s, t$ in the domain of $g$. It follows that $g$ is uniformly continuous on its domain, and so extends to a uniformly continuous mapping $g:[0, \infty) \rightarrow S \cup\{\widehat{x}\}$ such that (1) holds for all $s, t \in[0, \infty)$.

Now define a uniformly continuous mapping $h: \widehat{S} \rightarrow[0, \infty)$ by

$$
h(x) \equiv \frac{2}{L} \rho(x, \widehat{x}) .
$$

Then $g \circ h$ is a contraction mapping on $\hat{S}$, and therefore on $S$, and

$$
\hat{x}=g(0)=g(h(\hat{x}))=g \circ h(\hat{x}) .
$$

Hence $\hat{x}$ is the unique fixed point of $g \circ h$ in $\hat{S}$. Since $S$ has the contraction mapping property, $\hat{x} \in S$, so $\left(x_{n}\right)_{n \geqslant 1}$ converges.

\subsection{Brouwer's fixed point theorem}

For completeness, we give a constructive proof of the approximate Brouwer fixed point theorem, extended to uniformly sequentially continuous functions; for novelty we give a proof based on David Gale's proof from [15]. Before we do this we require a few more definitions.

Let $X$ be a metric space and let $f$ be a function from $X$ into $X$. If $\rho(x, f(x))<\varepsilon$, then $x$ is called an $\varepsilon$-fixed point. A function $f: X \rightarrow X$ has approximate fixed points if for each $\varepsilon>0$ there exists an $\varepsilon$-fixed point of $f$ in $X$. If every uniformly continuous function from $X$ into $X$ has approximate fixed points, then $X$ is said to have the approximate fixed point property.

Gale's proof of Brouwer's fixed point theorem uses a generalisation of the game of Hex. An $n$-dimensional Hex board of size $k$ consists of vertices $V=\{1, \ldots, k\}^{n}$ with edges between two vertices $x, y \in V$ if $^{5}\|x-y\|=1$ and either $x_{i} \leqslant y_{i}$ for each $i$ or $y_{i} \leqslant x_{i}$ for each $i$. Then $n$-dimensional Hex is an $n$-player game where players take turns to pick unclaimed vertices. A player gains an edge of the hex board if she owns the nodes at either end; player $i$ wins the game by connecting the two $i$-banks

$$
\begin{aligned}
& i \text {-bank } 1=\left\{\left(v_{1}, \ldots, v_{n}\right) \in V: v_{i}=0\right\}, \\
& i \text {-bank } 2=\left\{\left(v_{1}, \ldots, v_{n}\right) \in V: v_{i}=k\right\},
\end{aligned}
$$

with her edges. The 'Hex Theorem' of [15] (which, being finitely combinatorial, is fully constructive) says that any colouring of an $n$-dimensional Hex board with at most $n$ colours has a winner (for $n>2$ there may be more than one).

\footnotetext{
${ }^{5}$ We use $\|\cdot\|$ throughout to represent either the maximum norm or the supremum norm.
} 
Lemma 2 Let $f$ be a function from the unit hypercube $[0,1]^{n}$ into itself. Then for all $\varepsilon, \delta>0$ either there exists $x \in[0,1]^{n}$ such that $\rho(x, f(x))<\varepsilon$ or there exist $x, x^{\prime} \in[0,1]^{n}$ such that $\rho\left(x, x^{\prime}\right)<\delta$ and $\rho\left(f(x), f\left(x^{\prime}\right)\right)>\varepsilon$.

Proof Write

$$
f(x)=\left(f_{1}(x), \ldots, f_{n}(x)\right) ;
$$

Fixing $\varepsilon, \delta>0$, without loss of generality take $\delta<\varepsilon / 3$. Pick $N>0$ such that $1 / N<\delta$, and subdivide $[0,1]^{n}$ into an $n$-dimensional Hex board of size $N$. We partition the set $V$ of vertices of this Hex board into sets $C_{1}^{+}, C_{1}^{-}, \ldots, C_{n}^{+}, C_{n}^{-}$, and $B$ such that

$$
\begin{aligned}
& x \in C_{1}^{+} \quad \Rightarrow \quad f_{1}(x)-x_{1}>\frac{2 \varepsilon}{3} ; \\
& x \in C_{1}^{-} \Rightarrow \quad x_{1}-f_{1}(x)>\frac{2 \varepsilon}{3} ; \\
& \vdots \\
& x \in C_{n}^{+} \quad \Rightarrow \quad f_{n}(x)-x_{n}>\frac{2 \varepsilon}{3} ; \\
& x \in C_{n}^{-} \quad \Rightarrow \quad x_{n}-f_{n}(x)>\frac{2 \varepsilon}{3} ; \\
& x \in B \quad \Rightarrow \quad\|f(x)-x\|<\varepsilon .
\end{aligned}
$$

By the Hex theorem, either $B$ is inhabited, and there exists $x \in[0,1]$ such that $\rho(x, f(x))<\varepsilon$, or, as we may assume, there exists an $i$-path from $i$-bank 1 to $i$-bank 2 for some $1 \leqslant i \leqslant n$. Since no vertex of $C_{i}^{+}$has $i$-th coordinate 1 and no vertex of $C_{i}^{-}$has $i$-th coordinate 0 , such a path contains points from each set. Hence there exist adjacent vertices $x, x^{\prime}$ such that $x \in C_{i}^{+}$and $x^{\prime} \in C_{i}^{-}$. Then $\left\|x-x^{\prime}\right\|<\delta<\varepsilon / 3$, $f_{i}(x)>f_{i}\left(x^{\prime}\right)$, and

$$
\begin{aligned}
f_{i}(x)-f_{i}\left(x^{\prime}\right) & =\left(f_{i}(x)-x\right)+\left(x-x^{\prime}\right)+\left(x^{\prime}-f_{i}\left(x^{\prime}\right)\right) \\
& >\frac{2 \varepsilon}{3}-\frac{\varepsilon}{3}+\frac{2 \varepsilon}{3}=\varepsilon .
\end{aligned}
$$

Therefore $\rho\left(f(x), f\left(x^{\prime}\right)\right)>\left|f_{i}(x)-f_{i}\left(x^{\prime}\right)\right|>\varepsilon$.

With this lemma at hand we can weaken the standard hypothesis of the approximate Brouwer fixed point theorem; we only require that $f:[0,1]^{n} \rightarrow[0,1]^{n}$ be uniformly sequentially continuous ${ }^{6}$ : for all sequences $\left(x_{n}\right)_{n \geqslant 1},\left(y_{n}\right)_{n \geqslant 1}$ in $[0,1]^{n}$, if $\rho\left(x_{n}, y_{n}\right)$

\footnotetext{
${ }^{6}$ Throughout this paper, uniformly sequentially continuous can be substituted for uniformly continuous in the definition of the approximate fixed point property.
} 
tends to zero as $n \rightarrow \infty$, then $\rho\left(f\left(x_{n}\right), f\left(y_{n}\right)\right)$ also tends to zero as $n \rightarrow \infty$. It is easy to see that uniform continuity implies uniform sequential continuity; the converse cannot be proved constructively (see [7]).

Theorem 3 Let $f$ be a uniformly sequentially continuous function from the unit hypercube $[0,1]^{n}$ into itself. Then $f$ has approximate fixed points.

Proof We construct, using countable choice, sequences $\left(x_{n}\right)_{n \geqslant 1},\left(x_{n}^{\prime}\right)_{n \geqslant 1}$ as follows. For each $n \in \mathbf{N}$, apply Lemma 2 to construct either $x \in[0,1]^{n}$ such that $\rho(x, f(x))<\varepsilon$ or $x, x^{\prime} \in[0,1]^{n}$ such that $\rho\left(x, x^{\prime}\right)<1 / n$ and $\rho\left(f(x), f\left(x^{\prime}\right)\right)>\varepsilon$. In the latter case we set $x_{n}=x$ and $x_{n}^{\prime}=x^{\prime}$; in the former we set $x_{n}=x_{n}^{\prime}=x$. Then $\left(\rho\left(x_{n}, x_{n}^{\prime}\right)\right)_{n \geqslant 1}$ converges to zero. Since $f$ is uniformly sequentially continuous, there exists $N \in \mathbf{N}$ such that $\rho\left(f\left(x_{n}\right), f\left(x_{n}^{\prime}\right)\right)<\varepsilon$ for all $n \geqslant N$. It follows that $\rho\left(x_{N}, f\left(x_{N}\right)\right)<\varepsilon$.

Next we extend the approximate Brouwer fixed point theorem, for uniformly continuous functions, to compact convex subsets of $\mathbf{R}^{n}$. A subset $S$ of a normed space $X$ is strictly convex if for each $\varepsilon>0$ there exists $\delta>0$ such that for all $x, y$ in the boundary $\partial S$ of $S$, if ${ }^{7} \rho\left(\frac{1}{2}(x-y), \partial S\right)<\delta$, then $\|x-y\|<\varepsilon$. A normed space $X$ is uniformly convex if its unit ball is strictly convex: for all $\varepsilon>0$ there exists $\delta>0$ such that for all $x, y \in X$ with $\|x\|=\|y\|=1$, if $\left\|\frac{1}{2}(x-y)\right\|>1-\delta$, then $\|x-y\|<\varepsilon$. Any inner product space is uniformly convex [12, Page 93], and the $L_{p}$ spaces for $1<p<\infty$ are uniformly convex [4, Chapter 7, (3.22)].

Let $S$ be an inhabited subset of a metric space $X$, and let $x \in X$. We say that $b \in S$ is a closest point, or best approximation, to $x$ in $S$ if $\rho(x, b) \leq \rho(x, s)$ for all $s \in S$. The following extends Theorem 6 of [11].

Theorem 4 Let $S$ be a complete, located, convex subset of a uniformly convex normed space $X$. Then each point in $X$ has a unique closest point in $S$. Moreover, the mapping $Q$ from $X$ to $S$ sending $x$ to the best approximation to $x$ in $S$ is uniformly continuous.

Proof The proof of Theorem 6 in [11] establishes that for each $x \in X$ and each $\varepsilon>0$ the set

$$
S_{\varepsilon}^{x}=\{y \in S: \rho(x, y)<\rho(x, S)+\varepsilon\}
$$

\footnotetext{
${ }^{7}$ We do not require $\partial S$ to be located here: for an arbitrary subset $S$ of a metric space $X$ we use ' $\rho(x, S)<\varepsilon$ ' as a shorthand for 'there exists $s \in S$ with $\rho(x, s)<\varepsilon$. If $S$ is located, then this coincides with the standard meaning.
} 
has diameter no greater than $\varepsilon$, and hence that $x$ has a unique best approximation in $S$. To see that $Q$ is uniformly continuous, observe that if $\|x-y\|<\varepsilon / 2$, then $S_{\varepsilon / 2}^{y} \subset S_{\varepsilon}^{x}$. Hence $Q(x), Q(y) \in S_{\varepsilon}^{x}$, so $\|Q(x)-Q(y)\| \leqslant \varepsilon$.

We call the mapping $Q$ from the preceding theorem the projection onto $S$.

Theorem 5 Every totally bounded set $S$ of $\mathbf{R}^{n}$ with convex closure has the approximate fixed point property.

Proof Let $S$ be a subset of $\mathbf{R}^{n}$ satisfying the conditions of the theorem; without loss of generality we may assume that $S$ is both closed and a subset of the unit cube $[0,1]^{n}$. Fix $\varepsilon>0$ and let $Q$ be the projection mapping from $[0,1]^{n}$ onto $S$ (which exists, by the preceding theorem). Applying Theorem 3 to the mapping $f \circ Q:[0,1]^{n} \rightarrow[0,1]^{n}$, construct $x \in[0,1]^{n}$ such that $\|x-f \circ Q(x)\|<\varepsilon / 2$. Then

so

$$
\begin{aligned}
\|x-Q(x)\| & =\rho(x, S) \\
& \leqslant\|x-f \circ Q(x)\|<\frac{\varepsilon}{2},
\end{aligned}
$$

$$
\begin{aligned}
\|Q(x)-f(Q(x))\| & \leqslant\|Q(x)-x\|+\|x-f \circ Q(x)\| \\
& <\frac{\varepsilon}{2}+\frac{\varepsilon}{2}=\varepsilon .
\end{aligned}
$$

Hence $Q(x)$ is an $\varepsilon$-fixed point of $f$.

For a subset $S$ of a metric space $X$ we write

$$
S_{\varepsilon}=\{y \in X: \rho(x, y)<\varepsilon \text { for some } x \in S\} .
$$

Classically, Brouwer's fixed point theorem holds for any metric space which is homeomorphic to $[0,1]^{n}$; this also holds constructively. For subsets of uniformly convex normed spaces, this result is classically equivalent to, but seems constructively weaker than, the following.

Proposition 6 Let $X$ be a uniformly convex normed space, let $S$ be a subset of $X$ with the approximate fixed point property, and let $T$ be a subset of $X$ such that for each $\varepsilon>0$ there exists a uniformly bicontinuous ${ }^{8}$ function $f_{\varepsilon}: S \rightarrow T$ such that $f_{\varepsilon}(S)$ is convex and totally bounded and

$$
f_{\varepsilon}(S) \subset T \subset\left(f_{\varepsilon}(S)\right)_{\varepsilon} .
$$

Then $T$ has the approximate fixed point property.

\footnotetext{
${ }^{8}$ A function $f$ from $X$ onto $Y$ is uniformly bicontinuous if both $f$ and its inverse are uniformly continuous.
} 
Proof Let $f$ be a uniformly continuous function from $T$ into itself, and fix $\varepsilon>0$. Let $\delta>0$ be such that for all $x, y \in T$, if $\|x-y\|<\delta$, then

$$
\left\|f_{\varepsilon / 2}(x)-f_{\varepsilon / 2}(y)\right\|<\varepsilon / 2,
$$

where $f_{\varepsilon / 2}$ is as in the statement of the proposition. Let $Q$ be the projection onto $f_{\varepsilon / 2}(S)$ restricted to $T$, and let $I: f_{\varepsilon / 2}(S) \rightarrow T$ be the inclusion mapping; note that $\|Q(t)-t\|<\varepsilon / 2$ for all $t \in T$.

Then $f_{\varepsilon / 2}^{-1} \circ Q \circ f \circ I \circ f_{\varepsilon / 2}$ is a uniformly continuous function from $S$ into $S$. Hence there exists $x \in S$ such that

$$
\left\|f_{\varepsilon / 2}^{-1} \circ Q \circ f \circ f_{\varepsilon / 2}(x)-x\right\|<\delta .
$$

Then

$$
\left\|Q \circ f \circ f_{\varepsilon / 2}(x)-f_{\varepsilon / 2}(x)\right\|<\varepsilon / 2,
$$

and so

$$
\begin{aligned}
\left\|f\left(f_{\varepsilon / 2}(x)\right)-f_{\varepsilon / 2}(x)\right\| \leqslant & \left\|f \circ f_{\varepsilon / 2}(x)-Q \circ f \circ f_{\varepsilon / 2}(x)\right\| \\
& \quad+\left\|Q \circ f \circ f_{\varepsilon / 2}(x)-f_{\varepsilon / 2}(x)\right\| \\
\leqslant & \frac{\varepsilon}{2}+\frac{\varepsilon}{2}=\varepsilon .
\end{aligned}
$$

Thus $Q\left(f_{\varepsilon / 2}^{-1}(x)\right)$ is an $\varepsilon$-fixed point of $f$.

The Brouwer fixed point theorem for uniformly continuous functions is equivalent to the essentially nonconstructive lesser limited principle of omniscience,

LLPO: For each binary sequence $\left(a_{n}\right)_{n \geqslant 1}$, either $a_{n}=0$ for all even $n$ or $a_{n}=0$ for all odd $n$.

A straightforward modification of the Brouwerian example on page 8 of [4] shows that Brouwer's fixed point theorem implies LLPO. In order to see the converse, suppose that LLPO holds. Given a uniformly continuous function $f$ from a compact convex subset $S$ of $\mathbf{R}^{n}$ into itself, define a uniformly continuous function $g: S \rightarrow \mathbf{R}$ by $g(x)=\|x-f(x)\|$. By Theorem 5 the infimum of $g$ is zero. Since LLPO is equivalent (see [17]) to the principle

MIN: Every uniformly continuous mapping from a compact metric space into $\mathbf{R}$ attains its infimum. 
there exists $x \in S$ such that $g(x)=0 ; x$ is then a fixed point of $f$.

To obtain the usual conclusion of the intermediate value theorem, it suffices to assume that $f: \mathbf{R} \rightarrow \mathbf{R}$ is locally nonzero: for all $x \in \mathbf{R}$ and all $\varepsilon>0$ there exists $y \in(x-\varepsilon, x+\varepsilon)$ such that $f(y) \neq 0$. The equivalent notion for Brouwer's fixed point theorem, that $f: X \rightarrow X$ is locally John Doe-for all $x \in X$ and all $\varepsilon>0$ there exists $y \in B(x, \varepsilon)$ such that $f(y) \neq y$-is not sufficient to prove Brouwer's fixed point theorem in higher dimensions: Orevkov [20] has given an example of a continuous function from $[0,1]^{2}$ into itself with no fixed point. Further, Veldman [23] has shown that Brouwer's fixed point theorem for continuous functions with at most one fixed point is equivalent to Brouwer's fan theorem for detachable bars (see also [3]). (We say that $f$ has at most one fixed point if

$$
\max \{\rho(x, f(x)), \rho(y, f(y))\}>0
$$

whenever $x \neq y$.)

A function $f: X \rightarrow X$, where $X$ is a metric space, is nonexpansive if $\rho(f(x), f(y)) \leqslant$ $\rho(x, y)$ for all $x, y \in X$.

The standard Brouwerian example showing that Brouwer's fixed point theorem implies LLPO also shows that we cannot prove constructively that every nonexpansive mapping on $[0,1]$ has the fixed point property. ${ }^{9}$ Using a standard classical argument (see for example [22]), we can, however, show that such a mapping has approximate fixed points.

Proposition 7 Let $S$ be a bounded convex subset of a normed space $X$ and let $f$ be a nonexpansive mapping of $S$ into itself. Then $f$ has approximate fixed points.

Proof Since we are only interested in approximate fixed points we may replace $X$ by its completion $\hat{X}$, and $S$ by its closure in $\hat{X}$; we may also assume that $0 \in S$. Fix $\varepsilon>0$ and let $N>0$ be such that $S$ is contained in the ball of radius $N$ centered on 0 . Pick

\footnotetext{
${ }^{9}$ Classically, every continuous nonexpansive mapping on a bounded closed subset of a uniformly convex Banach space has the fixed point property. The standard classical proof for Hilbert spaces (see [22]) requires the statement that 'every convex bounded closed subset of a Hilbert space is weakly compact', which implies LPO. As a consequence it seems likely that the most general formulation of the nonexpansive fixed point theorem is not equivalent to LLPO; however, if we restrict ourselves to weakly compact subsets of a Hilbert space, then the nonexpansive fixed point theorem follows from MIN.
} 
$r \in(1-\varepsilon / N, 1)$, and let $x$ be the unique fixed point of the contraction mapping $r f$. Then

$$
\|f(x)-x\|=\|f(x)-r f(x)\|=(1-r)\|f(x)\| \leqslant(1-r) N<\varepsilon .
$$

Hence $x$ is an $\varepsilon$-fixed point.

\section{Schauder's fixed point theorem}

In this section we extend Brouwer's fixed point theorem by considering compact convex subsets of arbitrary Banach spaces; this gives us Schauder's fixed point theorem.

We call a located subset $S$ of a normed space $X$ projective if there exists a uniformly continuous projection function $Q$ of $X$ onto $S$ such that $\rho(x, Q(x))=\rho(x, S)$ for each $x$ in $X$. We give an approximate version of Schauder's fixed point theorem for projective sets.

Lemma 8 Let $S$ be a totally bounded subset of a metric space $X$, fix $\beta>\alpha>0$, and let $S^{\prime}$ be a convex set such that for each $x \in S$ there exists $x^{\prime} \in S^{\prime}$ such that $\rho\left(x, x^{\prime}\right)<\alpha / 2$. Then there exists a uniformly continuous function $P$ from $S$ into $S^{\prime}$ such that $\|P(x)-x\|<\beta$ for all $x \in S$.

Proof Let $\left\{x_{1}, \ldots, x_{n}\right\}$ be an $\alpha / 2$-approximation to $S$, and for each $1 \leqslant i \leqslant n$ pick $x_{i}^{\prime} \in S^{\prime}$ such that $\rho\left(x_{i}, x_{i}^{\prime}\right)<\alpha / 2$. Then for each $x \in S$ there exists $i$ such that $\rho\left(x, x_{i}^{\prime}\right)<\alpha$.

Let $f_{1}, \ldots, f_{n}$ be the uniformly continuous functions from $S$ into $\mathbf{R}$ given by

$$
f_{i}(x)=\max \left\{0, \gamma-\left\|x-x_{i}^{\prime}\right\|\right\},
$$

where $\gamma=(\alpha+\beta) / 2$. Then for each $x \in S$, there exists $i$ such that

$$
f_{i}(x)>\gamma-\alpha
$$

whence

$$
P(x) \equiv \frac{\sum_{i=1}^{n} f_{i}(x) x_{i}^{\prime}}{\sum_{i=1}^{n} f_{i}(x)}
$$

defines a uniformly continuous map from $S$ into $S^{\prime}$. 
Let $r>0$ and write $\{1, \ldots, n\}$ as the disjoint union of two sets $P, Q$ such that

$$
\begin{aligned}
& i \in P \quad \Rightarrow \quad\left\|x-x^{\prime}\right\|<\gamma+r \\
& i \in Q \Rightarrow\left\|x-x^{\prime}\right\|>\gamma .
\end{aligned}
$$

Then $P(x)$ is a convex combination of points in $P$, so

$$
\|P(x)-x\| \leqslant \max \left\{\left\|x-x_{i}^{\prime}\right\|: i \in P\right\}<\gamma+r .
$$

Since $r>0$ is arbitrary, it follows that $\|P(x)-x\| \leqslant \gamma<\beta$ for all $x \in S$.

Theorem 9 Let $S$ be an inhabited, totally bounded, projective subset of a normed space $X$. Then $S$ has the approximate fixed points property.

Proof Let $f: S \rightarrow$ be a uniformly continuous function. Fixing $\varepsilon>0$, let $\left\{x_{1}, \ldots, x_{n}\right\}$ be an $\varepsilon / 8$-approximation to $S$. Using [4, Lemma 2.5, Chapter 7], construct a finitedimensional subspace $V$ of $X$, with a basis contained in $S$, such that

$$
\rho\left(x_{i}, V\right)<\varepsilon / 8
$$

for all $i \in\{1, \ldots, n\}$. For each such $i$ pick $x_{i}^{\prime} \in V$ such that $\left\|x_{i}-x_{i}^{\prime}\right\|<\varepsilon / 8$. Then for each $x \in S$, there exists $i \in\{1, \ldots, n\}$ such that $\left\|x-x_{i}^{\prime}\right\|<\varepsilon / 4$. Let $S$ be the closed convex hull of $\left\{x_{1}^{\prime}, \ldots, x_{n}^{\prime}\right\}$, and let $Q: S^{\prime} \rightarrow S$ be the restriction to $S^{\prime}$ of the projection onto $S$. If $\sum_{i=1}^{n} \lambda_{i}=1$ and each $\lambda_{i} \geq 0$, then

$$
\begin{aligned}
\left\|Q\left(\sum_{i=1}^{n} \lambda_{i} x_{i}^{\prime}\right)-\sum_{i=1}^{n} \lambda_{i} x_{i}^{\prime}\right\| & \leq \sum_{i=1}^{n} \lambda_{i}\left\|Q x_{i}^{\prime}-x_{i}^{\prime}\right\| \\
& =\sum_{i=1}^{n} \lambda_{i} \rho\left(x_{i}^{\prime}, S\right) \\
& \leq \sum_{i=1}^{n} \lambda_{i}\left\|x_{i}^{\prime}-x_{i}\right\|<\frac{\varepsilon}{8} ;
\end{aligned}
$$

thus $\|Q(x)-x\|<\varepsilon / 4$ for all $x \in S^{\prime}$.

Using Lemma 8, construct a uniformly continuous function $P: S \rightarrow S^{\prime}$ such that $\|P(x)-x\|<\varepsilon / 3$ for all $x \in S$. Then $P \circ f \circ Q$ is a uniformly continuous map from $S^{\prime}$ into $S^{\prime}$; by Brouwer's fixed point theorem, Theorem 3, there exists $x^{\prime} \in S^{\prime}$ such that

$$
\left\|P \circ f \circ Q\left(x^{\prime}\right)-x^{\prime}\right\|<5 \varepsilon / 12
$$


write $x=Q\left(x^{\prime}\right)$. Then $x \in S$ and

$$
\begin{aligned}
\|f(x)-x\| & \leqslant\left\|f(x)-P \circ f \circ Q\left(x^{\prime}\right)\right\|+\left\|P \circ f \circ Q\left(x^{\prime}\right)-x^{\prime}\right\|+\left\|x^{\prime}-x\right\| \\
& =\|f(x)-P(f(x))\|+\left\|P \circ f \circ Q\left(x^{\prime}\right)-x^{\prime}\right\|+\left\|x^{\prime}-Q\left(x^{\prime}\right)\right\| \\
& <\varepsilon / 3+5 \varepsilon / 12+\varepsilon / 4=\varepsilon .
\end{aligned}
$$

Hence $x$ is an $\varepsilon$-fixed point of $f$.

By Theorem 4, every complete, located, convex subset of a uniformly convex space is projective; this gives us the following result.

Corollary 10 Let $S$ be an inhabited, totally bounded subset of a uniformly convex normed space $X$ such that the closure $\bar{S}$ of $S$ is convex. Then $S$ has the approximate fixed points property.

Proof Since we are interested in approximate fixed points, replacing $X$ with its completion $\hat{X}$ and $S$ with its closure in $\hat{X}$, we may assume that $S$ is compact and convex. The result then follows from Theorems 4 and 9.

Strictly convex sets are also projective; the proof is similar to that of Theorem 4 (which, in turn, is based on the proof of Theorem 6 of [11]).

Theorem 11 Let $S$ be an inhabited, complete, located, strictly convex subset of a normed space $X$. Then each point in $X$ has a unique closest point in $S$. Moreover, the mapping $Q$ from $X$ to $S$ sending $x$ to the best approximation to $x$ in $S$ is uniformly continuous.

Proof Let $x$ be a point of $X$, fix $\varepsilon>0$, and let $\delta \in(0, \varepsilon / 2)$ be such that for all $x, y \in \partial S$, if $\rho\left(\frac{1}{2}(x-y), \partial S\right)<2 \delta$, then $\|x-y\|<\varepsilon / 2$. Set

$$
S_{\varepsilon}^{x}=\{y \in S:\|x-y\|<\rho(x, S)+\delta / 2\} ;
$$

and fix $y_{1}, y_{2} \in S_{\varepsilon}^{x}$. Either $\rho(x, S)<\delta / 2$ and

$$
\left\|y_{1}-y_{2}\right\| \leqslant\left\|y_{1}-x\right\|+\left\|y_{2}-x\right\|<\delta+\delta<\varepsilon,
$$

or, as we may assume, $\rho(x, S)>0$. Since $S$ is located $S \cup \sim S$ is dense in $X$; whence we can apply Proposition 5.15 of [12] to construct the unique points $y_{1}^{\prime}, y_{2}^{\prime}$ such that $y_{i}^{\prime}$ is in the intersection of

$$
\left[x, y_{i}\right] \equiv\{t x+(1-t) y: t \in[0,1]\}
$$


and $\partial S$. Then, for $i=1,2$,

$$
\rho\left(y_{i}, y_{i}^{\prime}\right)=\rho\left(x, y_{i}\right)-\rho\left(x, y_{i}^{\prime}\right)<\rho(x, S)+\delta / 2-\rho(x, S)=\delta / 2,
$$

so

$$
\begin{aligned}
\left\|x-\frac{1}{2}\left(y_{1}^{\prime}+y_{2}^{\prime}\right)\right\| & \leqslant \frac{1}{2}\left\|x-y_{1}^{\prime}\right\|+\frac{1}{2}\left\|x-y_{2}^{\prime}\right\| \\
& \leqslant \frac{1}{2}\left(\left\|x-y_{1}\right\|+\left\|y_{1}-y_{1}^{\prime}\right\|+\left\|x-y_{2}\right\|+\left\|y_{2}-y_{2}^{\prime}\right\|\right) \\
& <\rho(x, S)+2 \delta .
\end{aligned}
$$

Apply Proposition 7.15 of [12] again to construct the unique point $z$ in the intersection of $\left[x, \frac{1}{2}\left(y_{1}^{\prime}, y_{2}^{\prime}\right)\right]$ and the boundary of $S$. Then

$$
\begin{aligned}
\rho\left(z, \frac{1}{2}\left(y_{1}^{\prime}+y_{2}^{\prime}\right)\right) & =\rho\left(x, \frac{1}{2}\left(y_{1}^{\prime}+y_{2}^{\prime}\right)\right)-\rho(x, z) \\
& <\rho(x, S)+2 \delta-\rho(x, S)=2 \delta .
\end{aligned}
$$

Therefore, by our choice of $\delta,\left\|y_{1}^{\prime}-y_{2} \prime\right\|<\varepsilon / 2$, and so

$$
\begin{aligned}
\rho\left(y_{1}, y_{2}\right) & \leqslant \rho\left(y_{1}, y_{1}^{\prime}\right)+\rho\left(y_{1}^{\prime}, y_{2}^{\prime}\right)+\rho\left(y_{2}^{\prime}, y_{2}\right) \\
& <\delta / 2+\varepsilon / 2+\delta / 2<\varepsilon .
\end{aligned}
$$

Hence the diameter of $S_{\varepsilon}^{x}$ is no greater than $\varepsilon$. The proof then proceeds as in Theorem 4.

Corollary 12 Let $S$ be an inhabited, totally bounded, strictly convex subset of a normed space $X$ such that the closure of $S$ is strictly convex. Then $S$ has the approximate fixed points property.

Proof As for Corollary 8 .

In the proof of Theorem 9, we begin by approximating the convex, totally bounded set $S$ by a set $S^{\prime}$ contained in a finite dimensional subspace of $X$. We then use $f$ to define a uniformly continuous map from $S^{\prime}$ into itself to which we can apply Brouwer's fixed point theorem. In particular, this requires us to construct a uniformly continuous map from $S^{\prime}$ into $S$ which is close to the identity map; it is in order to construct this mapping that we require $S$ to be projective. In the following result we circumvent this requirement: by considering only open sets, we can ensure that $S^{\prime}$ is contained in $S$; we can then produce a uniformly continuous function from $S^{\prime}$ into $S^{\prime}$ by restricting the domain of $f$, rather than composing $f$ with a mapping from $S^{\prime}$ into $S$, as in Theorem 9. 
Theorem 13 Every inhabited, open, totally bounded, convex subset of a normed space has the approximate fixed points property.

Proof The proof is similar to that of the preceding theorem. Let $S$ be an inhabited, open, totally bounded, convex subset of a normed space $X$, and let $f: S \rightarrow S$ be a uniformly continuous function. Let $\left\{x_{1}, \ldots, x_{n}\right\}$ be an $\varepsilon / 6$-approximation to $S$. We construct, as follows, a finite-dimensional subspace $V$ of $X$ such that $V$ contains an $\varepsilon / 3$-approximation $\left\{x_{1}^{\prime}, \ldots, x_{n}^{\prime}\right\}$ to $S$. Let $V_{1}=\operatorname{span}\left\{x_{1}\right\}$ and $x_{1}^{\prime}=x_{1}$. Suppose that we have constructed $V_{k-1}$ and $x_{1}^{\prime}, \ldots, x_{k}^{\prime}$ and let $r \in(0, \varepsilon / 6)$ be such that $B\left(x_{k}, r\right) \subset S$. Either $\rho\left(x_{k}, V_{k-1}\right)>0$ or $\rho\left(x_{k}, V_{k-1}\right)<r$. In the first case we set

$$
V_{k}=\operatorname{span}\left\{V_{k-1}, x_{k}\right\}
$$

and $x_{k}=x_{k}^{\prime}$. In the second case, pick $x_{k}^{\prime} \in V$ such that $\left\|x_{k}-x_{k}^{\prime}\right\|<r$ and set $V_{k}=V_{k-1}$. Set $V=V_{n}$; it is easy to see that $\left\{x_{1}^{\prime}, \ldots, x_{n}^{\prime}\right\}$ is an $\varepsilon / 3$-approximation to $S$.

Let $S^{\prime}$ be the convex hull of $\left\{x_{1}^{\prime}, \ldots, x_{n}^{\prime}\right\}$. Then $S^{\prime} \subset S$ and, by Lemma 8 , there exists a uniformly continuous function $P: S \rightarrow S^{\prime}$ such that $\|P(x)-x\|<\varepsilon / 2$ for all $x \in S$. Using Brouwer's fixed point theorem, applied to $\left.P \circ f\right|_{S^{\prime}}: S^{\prime} \rightarrow S^{\prime}$, construct $x \in S^{\prime}$ such that $\|P \circ f(x)-x\|<\varepsilon / 2$. Then

$$
\begin{aligned}
\|f(x)-x\| & \leqslant\|f(x)-P \circ f(x)\|+\|P \circ f(x)-x\| \\
& <\varepsilon / 2+\varepsilon / 2=\varepsilon .
\end{aligned}
$$

Hence $x$ is an $\varepsilon$-fixed point of $f$.

We can extend Theorem 9 to give an approximate version of Rothe's theorem [21, 22] for projective sets.

Theorem 14 Let $S$ be a compact, convex, projective subset of a normed space $X$, and let $f$ be a uniformly continuous function from $S$ into $X$ which maps the boundary of $S$ into $S$. Then $f$ has approximate fixed points.

Proof Fix $\varepsilon>0$, let $Q$ be the projection onto $S$, and let $\delta \in(0, \varepsilon / 4)$ be such that if $\|x-y\|<\delta$, then $\|f(x)-f(y)\|<\varepsilon$. Since $Q \circ f$ is a uniformly continuous function from $S$ into $S$, it follows from Schauder's fixed point theorem for projective sets that there exists $x \in S$ such that $\|Q \circ f(x)-x\|<\delta$. Suppose that $\rho(f(x), S)>\varepsilon / 4$. Then $f(x) \notin S$ and $\rho(x, y) \geqslant \delta$ for all $y \in \partial S$-this contradicts that $\|Q \circ f(x)-x\|<\delta$. 
Therefore $\rho(f(x), S) \leqslant \varepsilon / 4$, so

$$
\begin{aligned}
\|f \circ Q(x)-Q(x)\| \leqslant & \|f \circ Q(x)-f(x)\|+\|f(x)-Q \circ f(x)\| \\
& +\|Q \circ f(x)-x\|+\|x-Q(x)\| \\
< & \varepsilon / 4+\varepsilon / 4+\delta+\delta<\varepsilon .
\end{aligned}
$$

Hence $Q(x)$ is an $\varepsilon$-fixed point of $f$.

Since Schauder's fixed point theorem implies Brouwer's fixed point theorem and follows from MIN, it is equivalent to LLPO.

\section{An application}

We give an application of the approximate Schauder fixed point theorem for uniformly convex spaces (Corollary 10). A standard application of Schauder's fixed point theorem is in proving Peano's Theorem asserting the existence of solutions to particular differential equations:

Peano's Theorem Let $A$ be a closed subset of $\mathbf{R}$, let $\left(x_{0}, y_{0}\right) \in A$, and let $r>0$ be such that if $\left|x-x_{0}\right| \leqslant r$ and $\left|y-y_{0}\right| \leqslant r$, then $(x, y) \in A$. Let $f: A \rightarrow \mathbf{R}$ be continuous, let

$$
M \geqslant \sup \left\{|f(x, y)|:\left|x-x_{0}\right| \leqslant r,\left|y-y_{0}\right| \leqslant r\right\},
$$

and set $h=\min \{r, r / M\}$. Then the differential equation

$$
y^{\prime}=f(x, y), y\left(x_{0}\right)=y_{0}
$$

has a solution $y$ on the interval $\left[x_{0}-h, x_{0}+h\right]$.

However, since the exact version of Peano's Theorem is constructively equivalent to LLPO (see [6], which also gives an alternative constructive proof of an approximate Peano's Theorem, [2] gives a proof that Peono's Theorem implies LLPO), we can only hope to prove an approximate version of Peano's Theorem.

There is another, more pressing, problem: Peano's Theorem asserts the existence of solutions to particular differential equations in the normed space $\mathcal{C}(I)$, for some interval $I$, with the supremum norm, but this normed space is not uniformly convex. To overcome this difficulty, we first approximate the sup norm with a uniformly convex 
norm - this relies on being able to restrict the possible solutions of (2) to a sufficiently friendly subset of $\mathcal{C}(I)$.

A solution to the differential equation (2) on an interval $I$ in $\mathbf{R}$ is precisely a fixed point of the mapping $U: \mathcal{C}(I) \rightarrow \mathcal{C}(I)$ given by

$$
U(y)=y_{0}+\int_{x_{0}}^{x} f(t, y(t)) d t .
$$

The differential equation (2) is said to have approximate solutions on an interval $I$ if for all $\varepsilon>0$ there exists a continuous function $y: I \rightarrow \mathbf{R}$ such that $\|U(y)-y\|<\varepsilon$.

To prove a constructive version of Peano's theorem, we need the following lemma. A subset $S$ of $\mathcal{C}(I)$ is Lipschitz if there exists $M>0$ such that for all $y \in S$ and all $x_{1}, x_{2} \in I$ we have

$$
\left|y\left(x_{1}\right)-y\left(x_{2}\right)\right| \leqslant M\left|x_{1}-x_{2}\right|
$$

that is, $M$ is a Lipschitz constant for each $y \in S$. We call $M$ a Lipschitz constant for $S$.

Lemma 15 If $S$ is a bounded Lipschitz subset of $\mathcal{C}(I)$, then for each $\varepsilon>0$ there exists $p>1$ such that $\left|\|y\|-\|y\|_{p}\right|<\varepsilon$ for all $y \in S$.

Proof Fix $\varepsilon>0$ and let $N$ be a bound for $S$ and $M$ be a Lipschitz constant for $S$. It suffices to choose $p>1$ such that $\left|\|y\|-\|y\|_{p}\right|<\varepsilon$, where $y$ is given by

$$
y(x)=\max \left\{M, \frac{4 N}{b-a}\right\}\left(1-\frac{2}{b-a}\left|x-\frac{a+b}{2}\right|\right)-N .
$$

This is possible because, in $\mathcal{C}([-1,1])$,

$$
\begin{aligned}
\|1-|x|\|_{p} & =\left(\int_{-1}^{1}|1-| x||^{p} d t\right)^{1 / p} \\
& =\left(2 \int_{0}^{1}|1-x|^{p} d t\right)^{1 / p} \\
& =\left(\frac{2}{p+1}\right)^{1 / p} \longrightarrow 1
\end{aligned}
$$

as $p \rightarrow \infty$.

Theorem 16 Let $A \subset \mathbf{R}^{2}$ be closed, $\left(x_{0}, y_{0}\right) \in A^{\circ}$, and $r>0$ be such that if $\left|x-x_{0}\right| \leqslant r$, then $(x, y) \in A$. Let $f: A \rightarrow \mathbf{R}$ be uniformly continuous, let

$$
M \geqslant \sup \left\{|f(x, y)|:\left|x-x_{0}\right| \leqslant r,\left|y-y_{0}\right| \leqslant r\right\},
$$


and let $h=\min \{r, r / M\}$. Then the differential equation

$$
y^{\prime}=f(x, y), \quad y\left(x_{0}\right)=y_{0}
$$

has approximate solutions.

Proof Fix $\varepsilon>0$, let $I=\left[x_{0}-h, x_{0}+h\right]$, and set

$$
\mathcal{M}=\left\{y \in \mathcal{C}(I):\left|y(t)-y_{0}\right| \leqslant r \text { for all } t \in I\right\} .
$$

Since $f$ is uniformly continuous, $U$ is also uniformly continuous. Define

$$
\begin{aligned}
S=\{y \in \mathcal{M}:(\|U y\| & \left.\leqslant\left|y_{0}\right|+M h\right) \wedge \\
& \left.\left(\forall_{x_{1}, x_{2} \in I}\left|y\left(x_{1}\right)-y\left(x_{2}\right)\right| \leqslant M\left|x_{1}-x_{2}\right|\right)\right\} .
\end{aligned}
$$

Let $y \in \mathcal{M}$ and $t \in I$. Then

$$
\begin{aligned}
\left|U y(t)-y_{0}\right| & =\left|\int_{x_{0}}^{x} f(t, y(t)) d t\right| \leqslant M h \leqslant r, \\
\|U y\| & =\left|y_{0}+\int_{x_{0}}^{x} f(t, y(t)) d t\right| \leqslant\left|y_{0}\right|+M h, \text { and } \\
\left|U y\left(x_{1}\right)-U y\left(x_{2}\right)\right| & \leqslant\left|\int_{x_{1}}^{x_{2}} f(t, y(t)) d t\right| \\
& \leqslant M\left|x_{1}-x_{2}\right| .
\end{aligned}
$$

Hence $U$ maps $\mathcal{M}$ into $S$. By (a slight variation of) [4, (5.6) pg. 102], $S$ is compact; and, by Lemma 15, there exists $p>1$ such that

$$
\left|\|y\|-\|y\|_{p}\right|<\varepsilon / 2,
$$

for all $y \in S$. We can now apply the Schauder fixed point theorem to $\left.U\right|_{S}$ to construct a $y \in S$ such that $\|U y-y\|_{p}<\varepsilon / 2$. Then

$$
\begin{aligned}
\|U y-y\| & <\|U y-y\|_{p}+\varepsilon / 2 \\
& <\varepsilon / 2+\varepsilon / 2=\varepsilon,
\end{aligned}
$$

so $y$ is an $\varepsilon$-fixed point of $U$.

The above proof readily extends to a system

$$
\begin{aligned}
y_{1}^{\prime} & =f_{1}\left(y_{1}, \ldots, y_{n}, x\right) \\
y_{2}^{\prime} & =f_{2}\left(y_{1}, \ldots, y_{n}, x\right) \\
& \vdots \\
y_{n}^{\prime} & =f_{n}\left(y_{1}, \ldots, y_{n}, x\right)
\end{aligned}
$$

of linear ordinary differential equations. 


\section{References}

[1] PHG Aczel and M Rathjen, Notes on Constructive Set Theory, Report No. 40, Institut Mittag-Leffler, Royal Swedish Academy of Sciences, 2001; www.ml.kva.se/preprints/archive/2000-2001/2000-2001-40.pdf.

[2] M Beeson, Foundations of Constructive Mathematics: Metamathematical Studies, Springer, Berlin/Heidelberg/New York, 1985.

[3] J Berger and H Ishihara, Brouwer's fan theorem and unique existence in constructive analysis, Math. Log. Quart., 51(4) (2005), 360-364; doi: 10.1002/malq.200410038.

[4] E A Bishop and D S Bridges, Constructive Analysis, Grundlehren der Math. Wiss., 279, Springer-Verlag, Heidelberg, 1985.

[5] DS Bridges, A constructive Morse theory of sets, in: Mathematical Logic and its Applications (D. Skordev, ed.), Plenum Publishing Corp., 1987, New York, 61-79.

[6] D S Bridges, Constructive solutions of differential equations, preprint.

[7] D S Bridges, H Ishihara, P Schuster, and L Vita, Strong continuity implies uniform sequential continuity, Arch. Math. Logic 44 (2005) 887-895; doi: 10.1007/s00153005-0291-1.

[8] D S Bridges, I Loeb, Glueing continuous functions constructively, Arch. Math. Log. 49(5)(2010), 603-616 ; doi: 10.1007/s00153-010-0189-4.

[9] D S Bridges, F Richman, W H Julian, and R Mines, Extensions and Fixed Points of Contractive Maps in $\mathbf{R}^{n}$, J. of Math. Anal. and Appl., 165(2)(1992), 438-456; doi: 10.1016/0022-247X(92)90050-N.

[10] D S Bridges and F Richman, Varieties of Constructive Mathematics, London Math. Soc. Lecture Notes 97, Cambridge Univ. Press, 1987.

[11] D S Bridges, F Richman, and P Schuster, Linear independence without choice, Ann. Pure Appl. Logic 101(2000), 95-102; doi: 10.1016/S0168-0072(99)00030-5.

[12] D S Bridges and L S Vîţă, Techniques of Constructive Analysis, Universitext, SpringerNew-York, 2006.

[13] J M Borwein, Completeness and the Contraction Principle, Proceedings of the American Mathematical Society, 87(2)(1983), 246-250; doi: 10.2307/2043697.

[14] HM Friedman, Set Theoretic Foundations for Constructive Analysis, Ann. Math. 105(1)(1977), 1-28; doi: 10.2307/1971023.

[15] D Gale, The game of hex and the Brouwer fixed-point theorem, American Mathematical Monthly, December 1979, 818-827; doi: 10.2307/2320146.

[16] M Hendtlass, Kakutani's fixed point theorem in constructive mathematics, submitted for publication. 
[17] H Ishihara, An omniscience principle, the König lemma and the HahnBanach theorem, Z. Math. Logik Grundlagen Math. 36(1990), 237 - 240; doi: 10.1002/malq.19900360307.

[18] U Kohlenbach, Applied Proof Theory: Proof Interpretations and their Use in Mathematics, Springer Monographs in Mathematics, 2008.

[19] J Myhill, Constructive set theory, J. Symbolic Logic 40(3) (1975), 347-382; doi: $10.2307 / 2272159$.

[20] V P Orevkov, A constructive map of the square into itself, which moves every constructive point, Dokl. Akad. Nauk SSSR 152(1963), 55-58.

[21] E Rothe, Zur Theorie der topologischen Ordnung und der Vektorfelder in Banachschen Räumen, Compos. Math. 5(1937), 177-196.

[22] D R Smart, Fixed Point Theorems, Cambridge Univ.Press, 1974.

[23] W Veldman, Brouwer's approximate fixed-point theorem is equivalent to Brouwer's fan theorem, in Logicism, Intuitionism, and Formalism, Part II, Synth. Libr., 341, Springer, Dordrecht, 2009, 277-299,; doi: 10.1007/978-1-4020-8926-8_14.

Department of Pure Mathematics, University of Leeds

Leeds LS2 9JT, UK

mmmrh@leeds.ac.uk

Received: 23 August $2011 \quad$ Revised: 13 February 2012 